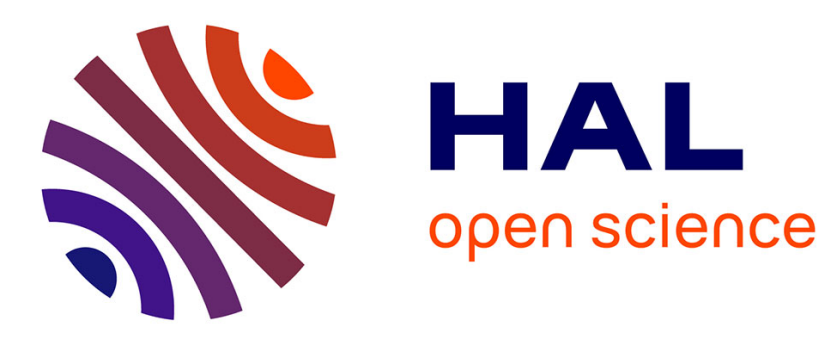

\title{
Liquid-liquid transition and critical point in sulfur
}

Laura Henry, Mohamed Mezouar, Gaston Garbarino, David Sifré, Gunnar

Weck, Frédéric Datchi

\section{To cite this version:}

Laura Henry, Mohamed Mezouar, Gaston Garbarino, David Sifré, Gunnar Weck, et al.. Liquid-liquid transition and critical point in sulfur. Nature, 2020, 584 (7821), pp.382-386. 10.1038/s41586-0202593-1 . hal-02954824

\section{HAL Id: hal-02954824 \\ https://hal.science/hal-02954824}

Submitted on 27 Nov 2020

HAL is a multi-disciplinary open access archive for the deposit and dissemination of scientific research documents, whether they are published or not. The documents may come from teaching and research institutions in France or abroad, or from public or private research centers.
L'archive ouverte pluridisciplinaire HAL, est destinée au dépôt et à la diffusion de documents scientifiques de niveau recherche, publiés ou non, émanant des établissements d'enseignement et de recherche français ou étrangers, des laboratoires publics ou privés. 


\section{Liquid-liquid transition and critical point in sulfur}

2 Laura Henry ${ }^{1}$, Mohamed Mezouar ${ }^{*}$, Gaston Garbarino ${ }^{1}$, David Sifré ${ }^{1}$, Gunnar Weck ${ }^{2}$ \&

3 Frederic Datchi ${ }^{3}$

$4 \quad{ }^{1}$ European Synchrotron Radiation Facility (ESRF), 71, Avenue des Martyrs, Grenoble,

5 France

$6 \quad{ }^{2}$ CEA, DAM, DIF, F-91297 Arpajon, France

$7 \quad{ }^{3}$ Institut de Minéralogie, de Physique des Milieux Condensés et de Cosmochimie

8 (IMPMC), Sorbonne Université, CNRS UMR 7590, MNHN, 4 place Jussieu, F-75005

9 Paris, France

10 A liquid-liquid transition (LLT) is a peculiar phenomenon in which a singlecomponent liquid transforms into another one via a first-order phase transition. Due to their counterintuitive nature, LLTs have intrigued scientists for several years and changed our perception of the liquid state. Such LLTs have been predicted from computer simulations of water ${ }^{1,2}$, silicon $^{3}$, carbon dioxide ${ }^{4}, \operatorname{carbon}^{5}$, hydrogen $^{6}$ and nitrogen ${ }^{7}$. Experimental evidence has been mostly found in supercooled, i.e. metastable, liquids such as $\mathrm{Y}_{2} \mathrm{O}_{3}-\mathrm{Al}_{2} \mathrm{O}_{3}$ mixtures ${ }^{8}$, water ${ }^{9}$ and other molecular liquids ${ }^{10,11,12}$, but the LLT in supercooled liquids often occurs simultaneously with crystallization, making it hard to separate the two phenomena ${ }^{13}$. A liquid-liquid critical point (LLCP), similar to the gas-liquid critical point, has been predicted at the end of the LLT line in some cases, but so far never experimentally observed for any materials. This putative LLCP has for instance been invoked in the case of water to understand its thermodynamic anomalies ${ }^{1}$. Here we report combined in-situ density measurements, $x$-ray diffraction and Raman scattering that provide direct evidence for a first-order liquid-liquid transition and a liquid-liquid critical point in sulfur. The transformation manifests itself by a sharp density jump between the low 
and high density liquids, and distinct features in the pair distribution function. A non-monotonic variation of the density jump with temperature is observed, which first increases on moving away from the critical point and then decreases. This behavior is linked to competing effects of density and entropy in driving the transition.

The pressure $(\mathrm{P})$-temperature $(\mathrm{T})$ phase diagram of sulfur exhibits important similarities with that of phosphorus which is to date the only element for which a direct in-situ observation of a LLT has been unambiguously evidenced ${ }^{14,15,16}$. The stable sulfur solids at room $\mathrm{P}, \alpha$ - and $\beta$-sulfur ${ }^{17,18}$, consist of $\mathrm{S}_{8}$ molecules, while at high $\mathrm{P}$-T, the stable polymorph is a polymeric solid composed of helical chains ${ }^{19}$. At room $\mathrm{P}$ the molecular character is conserved in the liquid upon melting at $388 \mathrm{~K}$ and up to $432 \mathrm{~K}$ where the socalled "lambda transition" $(\lambda-\mathrm{T})$ occurs $^{20}$. The $\lambda$-T has been described as a "living" polymerization transition ${ }^{21}$, reversible and incomplete (the polymer content reaches a maximum of $\sim 60 \%$ at the boiling point, $\mathrm{T}=718 \mathrm{~K}$ ), where a fraction of the $\mathrm{S}_{8}$ cyclic molecules open up and coalesce into long polymeric chains or rings. It is associated with

41 a large increase in viscosity and an anomalous but not discontinuous density variation ${ }^{20,22}$. For P above 5 GPa and T above $1000 \mathrm{~K}$, several P-T domains with different thermal and electrical properties have been proposed in liquid sulfur ${ }^{23}$. An experimental study ${ }^{24}$ suggested the existence above 6 GPa of a purely polymeric liquid composed of long chains below $1000 \mathrm{~K}$, which split to shorter chains at higher temperature. Ab-initio molecular dynamics simulations ${ }^{25}$ reproduced this chain breakage in the compressed

47 liquid but found no discontinuous change of density associated to this process. So far, no in-situ structural or vibrational studies have been conducted in the pressure region below

$493 \mathrm{GPa}$ in the mixed molecular-polymeric liquid. 
We performed in-situ x-ray absorption, x-ray diffraction and Raman scattering at the beamline ID27 of the European Synchrotron Radiation Facility (ESRF) ${ }^{26}$ to probe the density, structure and dynamics evolution of liquid sulfur in the P-T domain 0-3 GPa, 300-1100 K (see Supplementary Information S1-S3 for methods). The P-T paths are represented in the experimental phase diagram of sulfur in Fig. 1.

\section{Figure 1}

Density measurements were obtained in the Paris-Edinburgh press along eight isothermal (P1 to P8 in Fig. 1) and two isobaric pathways (P9 and P10). The accuracy of the density measured by this method is of the order of 1\% (Supplementary Information S1). XRD patterns of the sample were also collected at each P-T point to confirm that the sample was fully molten. As shown in Fig. 2a, along isothermal pathways P1-P5 below $1000 \mathrm{~K}$, we systematically observed a discontinuous jump in density over a very narrow pressure domain of $\sim 0.07 \mathrm{GPa}$, strongly suggesting the existence of a first-order phase transition between a low (LDL) and a high density liquid (HDL). Discontinuous density shifts were also observed upon varying the temperature at constant load along pathways P9-P10 (Supplementary Figure S9). These density jumps were accompanied by sudden changes in the structure factor $\mathrm{S}(\mathrm{Q})$ of the sulfur melt, as shown in Fig. 2b. This is particularly apparent on the width and position of the first diffraction peak which abruptly change at the transition. The density variation estimated from the measured S(Q) using the methodology of Ref. [27] (see Supplementary Information S3) very well compares with that derived from the x-ray absorption measurements (see inset of Fig. 2c), giving an independent confirmation of the discontinuous density jump at the transition. 
Fig. 2c presents x-ray radiographic images taken along a compression pathway at $74980 \mathrm{~K}$ and pressures between 1.6 and 2.5 GPa. These images show that below (panel $i$ ) and above (panel iv) the transition, the sample is homogeneous, while at the transition an interface separating a "bubble" of HDL from the surrounding LDL appears (panels ii and iii). As seen in the Supplementary video 1, the HDL bubble grows as the load is increased until the sample is fully in the HDL phase. These observations provide a compelling evidence of a coexistence of the LDL and HDL phases at the transition, and together with the density and structure factor measurements, confirm the first-order nature of the liquidliquid transition.

At $1090 \mathrm{~K}$ and $1100 \mathrm{~K}$ (pathways P7 and P8 in Fig. 1), we did not observe, within uncertainties, any discontinuous shift of the density as a function of pressure thus indicating the presence of a liquid-liquid critical point (LLCP). The LLCP was likely crossed along the pathway P6 at 2.15 GPa and 1035 K (indicated by a star in Fig. 2a) where the density measurements show clear anomalies (see Supplementary Information S2), while at lower and higher pressures along this isotherm, the density appears to continuously vary with pressure. As shown in Fig. 2d, we observe a non-monotonic evolution of the density discontinuity with temperature: starting from zero at the LLCP, it first increases to reach a maximum of $\sim 7.5 \%$ at about $750 \mathrm{~K}$ and then decreases.

\section{$91 \quad$ Figure 2}

Fig. 3a shows the pair distribution function (PDF) g(r) obtained by Fourier transform of the measured $\mathrm{S}(\mathrm{Q})$ at 5 selected points along the P8 pathway: A, B and C

94 are in the LDL domain, while D and E are in the HDL domain, close to the LDL-HDL transition line (see Fig. 1). The LDL PDF at point A (0.11 GPa, $428 \mathrm{~K})$ is very similar to

96 those reported for the ambient-pressure molecular liquid below the lambda transition 28,29 . 
97 It displays three well-defined peaks at 2.05, 3.39(2) and 4.45(2) $\AA$, in very good 98 agreement with the previous cited works. As shown in the latter, the third peak is a 99 fingerprint of the $S_{8}$ molecule since it occurs at the average distance of the third and fourth 100 neighbors in a $S_{8}$ ring, as deduced from the structure of the molecular $\alpha$-sulfur crystal. 101 When the temperature is increased in the LDL domain above the lambda transition (points $102 \mathrm{~B}$ at $0.17 \mathrm{GPa}-442 \mathrm{~K}$, and $\mathrm{C}$ at $0.36 \mathrm{GPa}-487 \mathrm{~K}$ ), the observed evolution is also very 103 similar to that described in the literature for the ambient-pressure liquid ${ }^{28}$. Namely, the 104 positions and intensities of the first and second-neighbor peaks are weakly affected, 105 whereas the third peak strongly reduces in intensity and becomes bi-modal. These 106 changes in the third/fourth neighbor distribution are a signature of the rapid increase of 107 polymer content and associated reduction of the $\mathrm{S}_{8}$ content above the lambda transition.

108 Indeed, the peak at $4.45 \AA$, characteristic of $\mathrm{S}_{8}$ molecules, reduces in intensity and a new

109 component at $4 \AA$ appears and grows with temperature, originating from the formation of 110 long polymeric chains or rings. The similarities between the present PDF in the region

111 from 4 to $5 \AA$ and those reported by Bellissent et al. ${ }^{28}$ from ambient-pressure neutron 112 diffraction can be appreciated from the comparison of the inset of Fig. 3a and Fig. 3b. We 113 note however that the new component in the ambient pressure PDF appears at a larger 114 distance, around $4.2 \AA$, which is likely due to the lower density of the ambient pressure 115 liquid.

\section{Figure 3}

118 We now come to the structural modifications in the PDF across the LDL-HDL 119 transition. As seen in Fig. 3c and S15, no change, within uncertainties, occurs on the first 120 and second peak positions, showing that the S-S bond length and the $<$ S-S-S $>$ angle are 
121 the same as in the LDL. The most important modifications occur again in the third/fourth122 neighbour distributions. The bi-modal shape of the third peak is maintained but the 123 component at $4.45 \AA$ is even more reduced, and the component located at $4 \AA$ in the LDL 124 undergoes a sudden shift in position to $4.15 \AA$ in the HDL. This shows that the local order 125 in the liquid changes at the transition, and further, suggests that the polymer content in 126 the HDL is larger than in the LDL. The latter point is confirmed by the comparison of the 127 Raman spectra measured in the LDL and HDL shown in Fig. 3d. In the HDL domain, we 128 observe an intensity increase of the stretching mode at $460 \mathrm{~cm}^{-1}$ assigned to the polymeric 129 chains $^{30}$, concomitant with a drop of the molecular bending mode at $152 \mathrm{~cm}^{-1}$ and 130 breathing mode at $220 \mathrm{~cm}^{-1}$ of the $\mathrm{S}_{8}$ molecule, attesting that the latter are residual in the 131 HDL region.

132 This work thus demonstrates that sulfur undergoes a first-order phase transition between two thermodynamically stable liquids, with clear experimental evidence of a

134 sharp density increase and structural modifications. We stress that this LLT is distinct 135 from the long-known lambda transition, which is associated to second-order like changes 136 in density ${ }^{20,22}$ and heat-capacity ${ }^{31}$. Furthermore, the lambda transition temperature slowly 137 decrease with pressure ${ }^{32}$ whereas for the present LLT, the transition temperature increases 138 with pressure (see also Supplementary Information S4). By virtue of the Clapeyron 139 equation, and since a positive jump of density occurs at the transition, this tells us that the 140 entropy of the HDL is smaller than that of the LDL, which contrasts with the entropy 141 increase in the LDL at the lambda transition ${ }^{33}$. The entropy reduction across the LLT may 142 be due in part to the increase in polymer content revealed by present experiments, and 143 associated reduction in the mixing entropy. But the observed changes in the PDF also 144 indicate that the local conformation of neighbor polymeric units is modified, certainly to 
145 a more compact arrangement imposed by the density increase, leading to a reduction in

146 the conformation entropy as well.

147 The shape of the transition line and the presence of a critical point make this LLT

148 in sulfur strongly resemble the well-known liquid-gas transition (LGT). However, there

149 is an important difference, which is the non-monotonic variation of the density jump with

150 temperature of the LLT, which first increases from zero as the temperature is decreased

151 from the LLCP and then decreases, contrasting with the monotonic increase of density

152 along the LGT transition. Such a behavior was recently predicted by AIMD calculations

153 along the LLT line of phosphorus ${ }^{34}$, from which the authors suggested that the order

154 parameter describing the LLT contains contributions from both the density and entropy,

155 and that at least at low temperatures, entropy governs the transition rather than density.

156 This is at odds with the LGT where density is the sole order parameter. A two-order

157 parameters model including density, on one hand, and a bond-order parameter describing

158 locally favoured structures, on the other hand, was also proposed by Murata and Tanaka ${ }^{13}$

159 to explain the existence of LLTs. The putative LLT in water has also been described as

160 entropy-driven based on a model where water is considered as an "athermal solution" of

161 two molecular structures with different entropies and densities ${ }^{35}$. We note however that

162 the present LLT in sulfur is different from that in water and phosphorus in the sense that

163 the transition line has a positive slope in sulfur but a negative one in water and

164 phosphorus. This may signal that sulfur belongs to a different class of LLT.

165 This work also provides the first, to the best of our knowledge, experimental 166 evidence for a critical point terminating the line of a LLT. Such a LLCP was proposed in 167 phosphorus at about $3500 \mathrm{~K}-0.02 \mathrm{GPa}^{34}$, conditions which have not yet been achieved 168 by experiment. In supercooled liquid silicon, classical empirical calculations have 
169 predicted a LLCP at negative pressures (-0.6 GPa, $1120 \mathrm{~K})^{36}$ but ab-initio calculations 170 recently determined that the HDL and HDL-vapor spinodals form a continuous reentrant 171 curve, making supecooled Si a critical point free system ${ }^{37}$. In water, the existence of a

172 LLCP has been conjectured by Poole in 1992 et al. ${ }^{1}$ to explain the many anomalies in the 173 thermodynamic properties such as the heat capacity, compressibility and thermal 174 expansion coefficients, and evidence for this LLCP has been strongly searched for and 175 debated ever since. The experimental observation of this hypothetical LLCP in water may 176 well never be possible as it is located in the "no man's land", i.e. the P-T domain below 177 the homogeneous nucleation temperature. Finally, a LLCP has also been predicted in 178 simple molecular systems, such as $\mathrm{H}_{2}{ }^{6}$ and $\mathrm{N}_{2}{ }^{7}$ but their experimental observation remain 179 extremely challenging. The LLCP in sulfur, being in a P-T range easily accessible by 180 experiment, provides a unique opportunity for the study of critical phenomena associated to LLTs. We thus expect that the present work will spark a new interest in liquid-liquid transitions that will provide a solid basis for understanding the principles that govern

183 LLTs in general. Future works should also focus on deciphering the microscopic 184 processes at the origin of the LLT in sulfur.

185

186

187

188

189

190

191

192 


\section{References}

1. Poole, P. H., Sciortino, F., Essmann, U. \& Stanley, H. E. Phase behaviour of metastable water. Nature 360, 324-328 (1992).

196

2. Harrington, S., Zhang, R., Poole, P. H., Sciortino, F. \& Stanley, H. E. Liquid-liquid phase transition: Evidence from simulations. Phys. Rev. Lett. 78, 2409- 2412 (1997).

3. Sastry, S. \& Angell, C. A. Liquid-liquid phase transition in supercooled silicon. Nature Mater. 2, 739-743 (2003).

4. Boates, B., Teweldeberhan, A. M., \& Bonev, S. A. Stability of dense liquid carbon dioxide. Proc. Natl. Acad. Sci. U. S. A. 109, 14808-14812 (2012).

5. Glosli, J. N. \& Ree, F. H. Liquid-liquid phase transformation in carbon. Phys. Rev. Lett. 82, 4659- 4662 (1999).

6. Morales, M. A., Pierleoni, C., Schwegler, E. \& Ceperley, D. M. Evidence for a first-order liquid-liquid transition in high-pressure hydrogen from ab initio simulations. Proc. Natl. Acad. Sci. USA 107, 12799 (2010).

7. Boates, B. \& Bonev, S. First-order liquid-liquid phase transition in compressed nitrogen. Phys. Rev. Lett. 102, 015701 (2009). system Al2O3-Y2O3. Nature 369, 633-636 (1994).

9. Mishima, O. \& Stanley, H. E. The relationship between liquid, supercooled and glassy water. Nature 396, 329-335 (1998). transition in supercooled aqueous solution related to the HDA-LDA transition. 
12. Kurita, R. \& Tanaka H. On the abundance and general nature of the liquid-liquid phase transition in molecular systems. J. Phys. Condens. Matter 17, 293-302 (2005).

13. Murata, K. \& Tanaka, H. Microscopic identification of the order parameter governing liquid-liquid transition in a molecular liquid. Proc. Natl. Acad. Sci. USA 112, 5956 (2015).

14. Katayama, Y., Mizutani, T., Utsumi, W., Shimomura, O., Yamakata, M. \& Funakoshi, K. A. First-order liquid-liquid phase transition in phosphorus. Nature, 403, 170-173, (2000).

15. Monaco, G., Falconi, S., Crichton, W. A. \& Mezouar, M. Nature of the first-order phase transition in fluid phosphorus at high temperature and pressure, Phys. Rev. Lett. 90, 255701 (2003).

16. Katayama, Y., Inamura, Y., Mizutani, T., Yamakata, M. , Utsumi, W. \& Shimomura, O. Macroscopic separation of dense fluid phase and liquid phase of phosphorus. Science 306, 848-851 (2004).

17. Steudel, R. \& Eckert, B. Solid sulfur allotropes. Top. Curr. Chem. 230, 1-79 (2003).

18. Templeton, L. K., Templeton, D. H. \& Zalkin, A. Crystal-structure of monoclinic sulfur. Inorg. Chem. 15, 1999-2001 (1976).

19. Crichton, W. A., Vaughan, G. B. M. \& Mezouar, M. In situ structure solution of helical sulfur at 3 GPa and 400C. Z. Kristallogr. 216, 417-419 (2001). (1967). 780-782 (1959). temperature. J. Chem. Phys. 96, 2175 (1992). 
23. Brazhkin, V. V., Popova, S. V. \& Voloshin, R. N. Pressure-temperature phase diagram of molten elements: selenium, sulfur and iodine. Physica B 265, 64-71 (1999).

24. Liu, L. Kono, Y., Kenney-Benson, C., Yang, W., Bi, Y., \& Shen, G. Chain breakage in liquid sulfur at high pressures and high temperatures. Phys. Rev. B 89, 174201 (2014).

25. Plašienka, D., Cifra, P. \& Martoňák R. Structural transformation between long and short-chain form of liquid sulfur from ab initio molecular dynamics. J. Chem. Phys. 142, 154502-512 (2015).

26. Mezouar, M. et al. Development of a new state-of-the-art beamline optimized for monochromatic single-crystal and powder X-ray diffraction under extreme conditions at the ESRF. J. Synchr. Rad. 12, 659-664(2005).

27. Eggert, J., Weck, G., Loubeyre, P. \& Mezouar M. Quantitative structure factor and density measurements of high-pressure fluids in diamond anvil cells by X-ray diffraction: Argon and water. Phys. Rev. B, 65, 174105 (2002).

28. Bellissent, R., Descotes, L., Boué, F. \& Pfeuty, P. Liquid sulfur: Local-order evidence of a polymerization transition. Phys. Rev. B, 41, 2135-2138, (1990).

29. Vahvaselka, K.S. \& Mangs, J.M. X-Ray diffraction study of liquid sulfur. Phys. Script. 38, 737-741, (1988).

30. Kalampounias, A. G., Kastrissios, D. T. \& Yannopoulos, S. N. Structure and vibrational modes of sulfur around the lambda-transition and the glass transition. $J$. Non Cryst. Sol. 326, 115-119 (2003).

31. Braune, H. \& Moller, O. The specific heat of liquid sulfur. Z. Naturforsch. 9a, 210-217 (1954). 
273

274

275

276

277

278

279

280

281

282

283

284

285

286

287

288

289

290

291

292

293

294

295

296

32. Kuballa, M. \& Schneider, G. Differential Thermal Analysis under High Pressure

I: Investigation of the Polymerisation of Liquid Sulfur. Berichte der Bunsengesellschaft für physikalische Chemie 75, 513-516 (1971).

33. Steudel, R. Liquid sulfur. Top. Curr. Chem. 230, 81-134 (2003).

34. Zhao, G., Wang, H., Hu, D. M., Ding, M. C., Zhao, X. G. \& Yan J. L. Anomalous phase behavior of first-order fluid-liquid phase transition in phosphorus. J. Chem. Phys. 147, 204501 (2017).

35. Holten, V. \& Anisimov, M.A. Entropy-driven liquid-liquid separation in supercooled water. Sci. Rep. 2, 713 (2012).

36. Vasisht, V. V., Saw, S. \& Sastry, S., Liquid-liquid critical point in supercooled silicon. Nat. Phys. 7, 549-553 (2011).

37. Zhao, G., Yu, Y.J., Yan, J.L., Ding, M.C., Zhao, X.G. \& Wang, H.Y. Phase behavior of metastable liquid silicon at negative pressure: Ab initio molecular dynamics. Phys. Rev. B 93, 140203 (2016). 
298 Fig. 1. Phase diagram of sulfur around the liquid-liquid transition. P1 to P8: isothermal pathways followed during the density measurements presented in Fig. 2. P1,

300 P2, P4 to P7 were made on compression while P3 (diamond symbols) and P7 (open black

301 circles) were made on decompression. For clarity, P7 and P8 are shown up to 3 GPa only.

302 P9 and P10 are isobaric pathways followed during the density measurements presented

303 in Fig. S9 (Supplementary Information S1). The letters A, B, C, D, E (blue filled triangles) 304 along path P11 indicate the P, T conditions of the selected XRD data in Fig. 3. I, II and

305 III are the P,T points of the Raman spectra presented in Fig. 3. The black dashed line is 306 the transition line between the low density liquid (LDL) domain (in yellow) and the high 307 density liquid (HDL) domain (in pink) that terminates at the critical point Cp (black solid 308 circle).

309 Fig. 2. First-order liquid-liquid transition in sulfur. a. Relative pressure variation of 310 the liquid density $\rho / \rho_{0}$ ( $\rho_{0}$ : density of the lowest pressure point for each isotherm) 311 collected along seven isothermal pathways (P1, P2 and P4 to P8 in Fig. 1). For clarity, 312 the density jump obtained on decompression along P3 and along the isothermal paths P9 313 and P10 are presented in the Extended Data Fig. 1 and 3. Main panel: at T below 1030 K, 314 a clear density jump is observed along all the isothermal paths. At 1035 K, a density 315 anomaly is detected in the vicinity of the LLCP (see Extended Data Fig. 4). Above the

316 LLCP (inset panel), a continuous variation of the density is observed. b. Structure factors $317 \mathrm{~S}(\mathrm{Q})$ of liquid sulfur collected along the isothermal path P2 ( $\mathrm{T}=650 \mathrm{~K})$. The red arrow 318 emphasizes the shift of the first peak position of S(Q) at the LDL-HDL transition. The $319 \mathrm{~S}(\mathrm{Q})$ 's collected on decompression at $740 \mathrm{~K}$ are shown in the Extended Data Fig. 2. The 320 variation of the density calculated from the associated Pair Distribution Function (PDF) 321 (black filled squares) is presented in the inset panel together with the one obtained from 322 the direct density measurements (red empty squares). c. X-ray radiography of liquid sulfur 323 across the LDL-HDL transition line at $\mathrm{T}=980 \mathrm{~K}$ and pressures between 1.6 and $2.5 \mathrm{GPa}$. 
324 i: Pure LDL, ii and iii: LDL-HDL coexistence, iv: Pure HDL. The yellow arrow indicates

325 the LDL-HDL boundary. d. Temperature evolution of the density jump. The black and

326 blue symbols correspond to the isothermal pathways (P1 to P8) and, the red symbols to

327 the isobaric (P9 and P10) pathways. A maximum of $7.5 \%$ is located at $\sim 750 \mathrm{~K}$.

328 Fig. 3. Local order in the low- and high-density liquid sulfur. a. The main panel 329 presents the pair distribution function $\mathrm{g}(\mathrm{r})$ of liquid sulfur at selected pressure and 330 temperature conditions along path P11 of Fig. 1. The letters A to E in parentheses refers 331 to the P,T conditions in Fig. 1 at which the measurements were performed. The curves 332 are vertically offset by 0.3 for clarity. The inset shows an enlargement of the PDF in the 333 region from 0.4 to $0.5 \mathrm{~nm}$ which contains contributions from third and fourth neighbours.

334 b shows an enlargement of the third peak of G(r) from the ambient-pressure neutron data 335 of Ref. 28 at 423 K, 473 K and 573 K (the curves were digitized from Fig. 2 of Ref. 28). 336 c presents the position of the second, third and fourth neighbours as a function of pressure, 337 as deduced from the peak positions of the PDF along the path P11. Panel $\mathbf{d}$ shows selected 338 Raman spectra of liquid sulfur collected at the P,T points I,II and III of Fig.1. I: LDL 339 below the $\lambda$-T. II: LDL above the $\lambda$-T. III: HDL.

\section{Extended Data Figure Legends}

341 Extended Data Fig 1. Density discontinuity at 740 K. Raw datasets of isothermal X-

342 ray absorption profiles I/I0 collected on decompression at $740 \mathrm{~K}$. The black arrow

343 indicates the density jump. B. Resulting isothermal density curve of sulfur in red and

344 density variation of $\mathrm{NaCl}$ pressure standard in blue.

345 Extended Data Fig 2. Structure factors S(Q) of liquid sulfur collected on

346 decompression along the isothermal path $\mathbf{T}=\mathbf{7 4 0} \mathrm{K}$. The black arrow emphasizes the

347 shift of the first peak position of S(Q) at the HDL-LDL transition. The variation of the

348 density calculated from the associated Pair Distribution Function (PDF) (black filled 
349 squares) is presented in the inset panel together with the one obtained from the direct

350 density measurements (red empty squares).

351 Extended data Fig 3. Isothermal density discontinuity. Density of liquid sulfur as a

352 function of temperature along isobaric paths P9 at $0.4 \mathrm{GPa}$ (left panel) and P10 at $1.3 \mathrm{GPa}$

353 (right panel).

354 Extended Data Fig 4. Liquid-liquid critical point in sulfur. Panels a and b: X-ray

355 absorption profiles I/I0 in the horizontal and vertical directions in the vicinity of the

356 critical point. Panels c and d: Horizontal x-ray absorption profiles at temperatures

357 below $(950 \mathrm{~K})$ and above $(1090 \mathrm{~K})$ the critical point. The red arrow in panel $\mathbf{c}$ indicates

358 the I/I0 discontinuity at the liquid-liquid transition. No I/I0 discontinuity is observed at

359 temperature above the critical point (panel d).

360

\section{Methods}

362 Methods are available in the Supplementary Information at https://XXX

364 Acknowledgments

365 The authors acknowledge the European Synchrotron Radiation Facility for provision of

366 synchrotron beamtime at the beamline ID27, the Agence Nationale de la Recherche for

367 financial support under Grant No. ANR 13-BS04-0015 (MOFLEX) and the company

368 Almax easy Lab for providing the diamond cylinders.

\section{Author contributions}

370 The original idea was defined by MM. The experiments were performed by LH, GG,

371 DS and MM with equal contributions. The data were analysed and the figures produced 
372 by LH with contributions from all the co-authors. The manuscript was written by MM

373 and FD with contributions from all the co-authors.

374 Correspondence and requests for materials should be addressed to M. M.

375 (mezouar@esrf.fr).

376 Data Availability

377 Tables containing the data points for all the main and extended data figures are provided

378 as Excel files. The data that support the findings of this study are available from the

379 corresponding author upon request.

380 Competing interests

381 The authors declare no competing interests. 


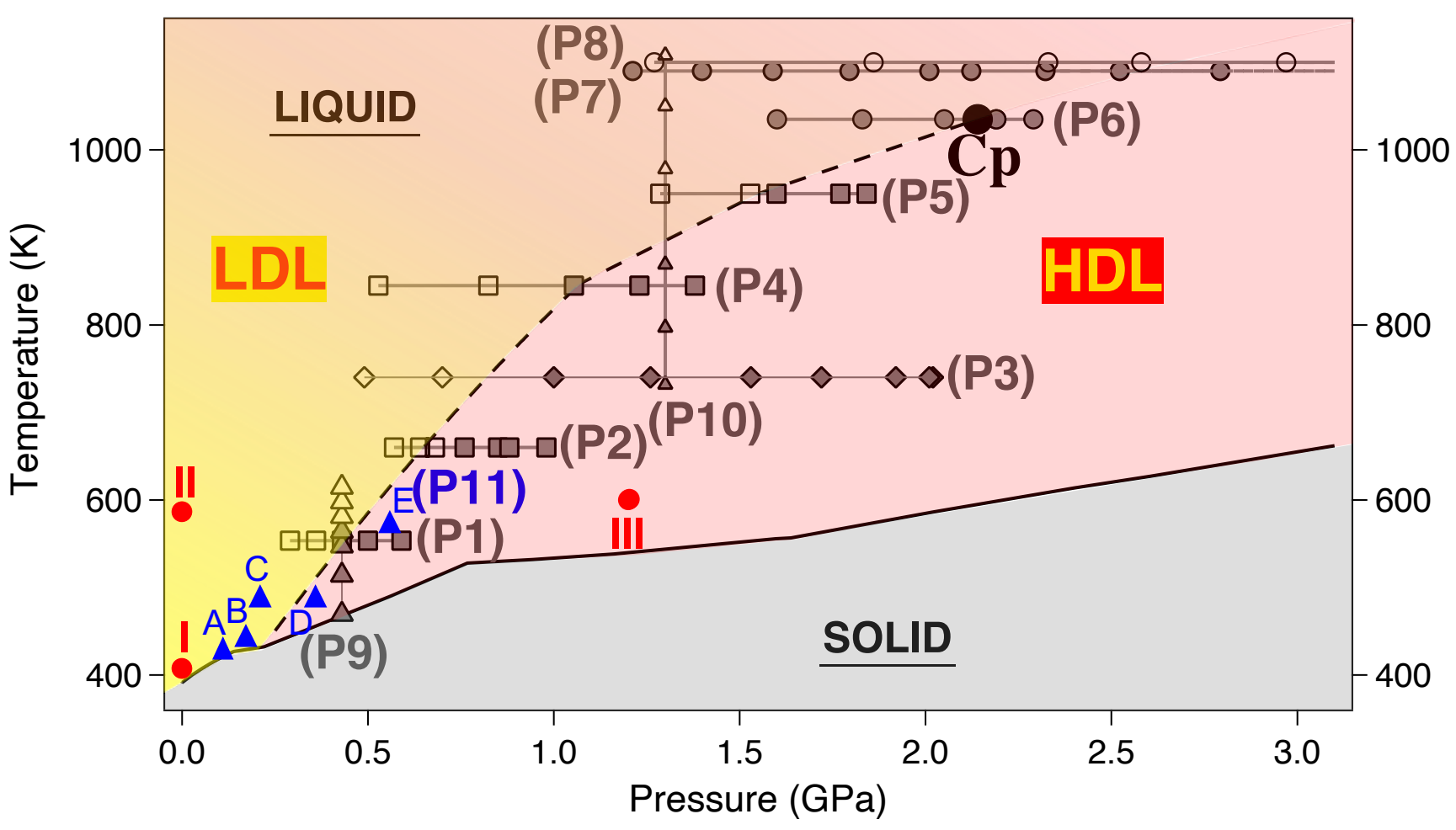




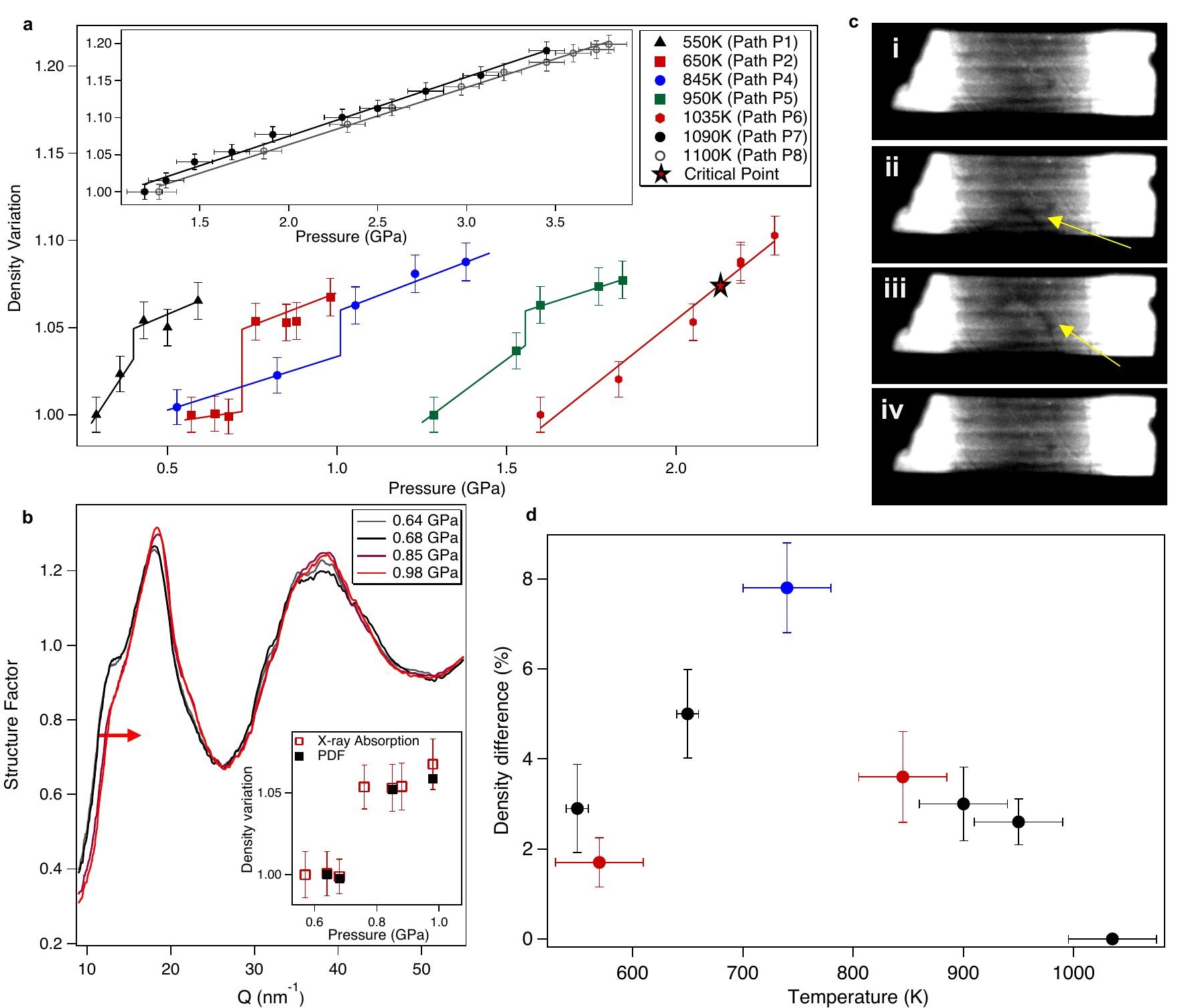



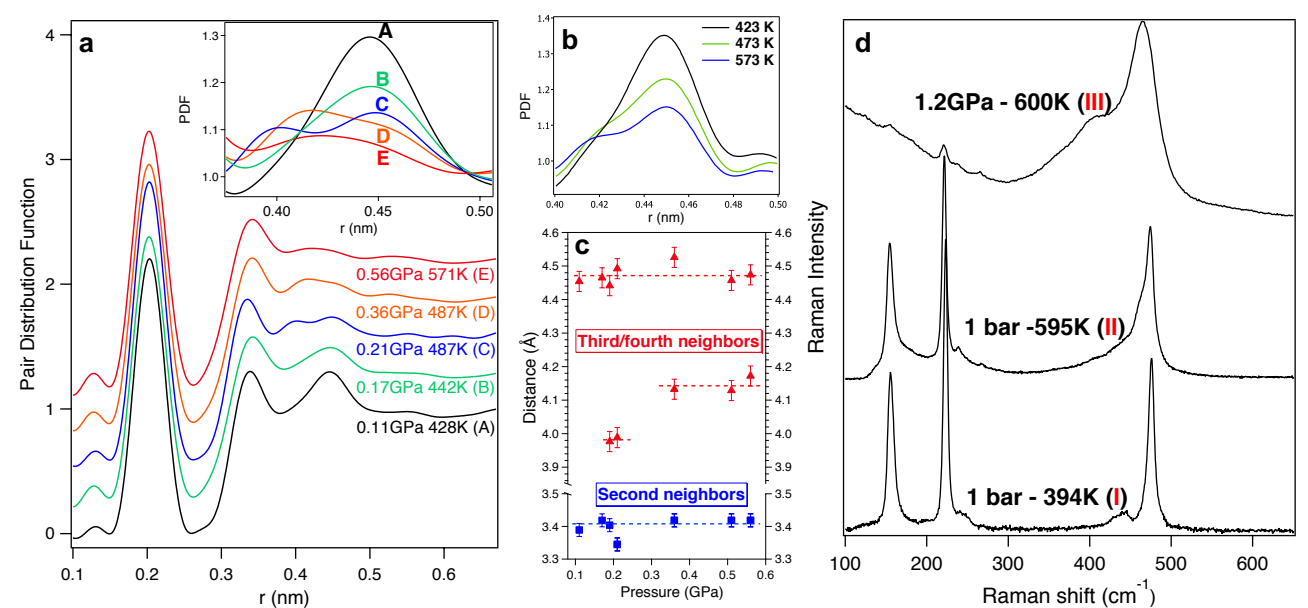